\title{
Coulisses
}

Revue de théâtre

2 | Automne 1990

Spécial Festival des idées : Besançon ville ouverte aux jeunes

\section{Marivaux sans marivaudage}

\section{Rédaction}

\section{(2) OpenEdition}

Journals

\section{Édition électronique}

URL : http://journals.openedition.org/coulisses/1608

DOI : 10.4000/coulisses. 1608

ISSN : 2546-9460

Éditeur

Presses universitaires de Franche-Comté

\section{Édition imprimée}

Date de publication : 1 novembre 1990

Pagination : $\mathrm{np}$

ISSN : 1150-594X

\section{Référence électronique}

Rédaction, «Marivaux sans marivaudage », Coulisses [En ligne], 2 | Automne 1990, mis en ligne le 04 juillet 2017, consulté le 23 octobre 2019. URL : http://journals.openedition.org/coulisses/1608 ; DOI : $10.4000 /$ coulisses. 1608

Ce document a été généré automatiquement le 23 octobre 2019.

Coulisses 


\section{Marivaux sans marivaudage}

\section{Rédaction}

1 Le Théâtre à tout prix, compagnie créée fin 1989, présente au cours de la même soirée mais en des lieux différents deux pièces de Marivaux : Les Fausses Confidences (PetitKursaal) et La Dispute (Grand-Kursaal). J.-M. Potiron, metteur en scène de ce spectacle, poursuit sa recherche sur le théâtre du XVIII ${ }^{e}$ siècle puisqu'il avait monté l'an dernier Le Mariage de Figaro de Beaumarchais.

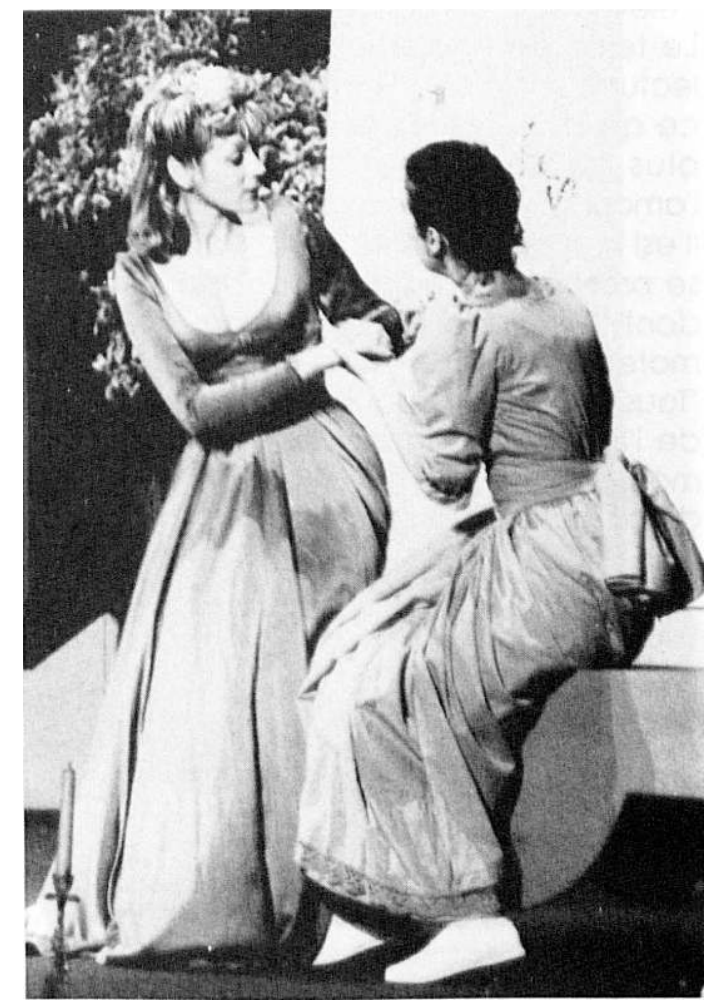

(C) Collectif Photo BVOJ 90. 


\section{Les Fausses Confidences}

\section{Une histoire d'amour?}

2 Dorante, jeune homme de bonne famille mais désargenté, s'introduit comme intendant chez Araminte, jeune veuve, jolie et très riche, dans le dessein de l'épouser, Il sera aidé dans son entreprise par Dubois, son ancien valet, fermement décidé à faire la fortune de Dorante :

Vous réussirez (dit-il à Dorante). Je m'en charge, je le veux, je l'ai mis là (geste vers sa tête). Nous sommes convenus de toutes nos actions; toutes nos mesures sont prises... Fierté, raison et richesse, il faudra que tout se rende. Quand l'amour parle, il est le maître, et il parlera.

3 Comme dans l'ensemble de son théâtre, Marivaux traite de l'amour. Côté Araminte, c'est le thème classique des surprises de l'amour: en l'espace de vingt-quatre heures, elle passe de l'indifférence à l'égard d'un inconnu à des sentiments très forts puisqu'elle épousera Dorante en dépit de la distance sociale et des pressions de son entourage. Quant à Dorante, il aime Araminte avec passion, ainsi qu'il l'affirme à Dubois.

Le spectateur s'attend donc à voir une belle histoire d'amour, en costume Ancien Régime, assaisonnée d'un zest de marivaudage, tradition oblige,

Au début, explique le metteur en scène, j'ai travaillé dans le sens de l'interprétation traditionnelle: Dorante aime Araminte, avec la figure classique valet-maîtremaîtresse où le valet fomente une intrigue pour que l'amour triomphe. Dubois est un manipulateur mais la cause est bonne s'il s'agit d'amour.

\section{Une histoire d'argent}

Dorante est-il poussé par l'amour ou aiguillonné par un intense désir d'ascension sociale? Et si les cinquante mille livres de rente d'Araminte étaient le mobile de l'action? La comédie prend alors une autre dimension, Des signes invitent le spectateur à suivre la piste de l'arrivisme. La blondeur lumineuse d'Araminte dans son éclatante robe rouge contraste avec la sombre silhouette de Dorante. Un indice plus sérieux est fourni par un élément central du décor: la porte. Cette porte, qui donne accès à l'appartement d'Araminte a une valeur stratégique et symbolique ; stratégique car c'est cette porte que Dorante rêve de pouvoir passer pour entrer dans la place; symbolique parce que la porte dans son architecture signifie les clivages sociaux. Elle présente en effet trois niveaux d'ouverture correspondant à l'échelle sociale :

- niveau bas que les valets empruntent en rampant

- moyen pour des passages roturiers

- élevé, pour de nobles entrées.

Le texte est également susceptible d'une lecture « sociale » :

Si on fait l'inventaire de ce qui est dit, précise J.-M. Potiron, il y a plus de choses sur l'arrivisme que sur l'amour.

7 Il est vrai que Dorante, dans son aveu final, se présente comme le jouet de son valet dont il souligne peu élégamment le rôle moteur :

Tous les incidents qui sont arrivés partent de l'industrie d'un domestique qui savait mon amour... (Il) m'a pour ainsi dire forcé de consentir à son stratagème. 

même, Les Fausses Confidences, est sujet à interprétation. Et si l'aveu de Dorante était une « vraie fausse confidence »? l'hypothèse : Dorante n'aime pas Araminte. Cependant, plutôt que de trancher, J.-M. Potiron a choisi de conserver l'ambiguïté du personnage et de ses motivations profondes :

Je n'offre pas de solution; je souhaite que le spectateur se pose la question.

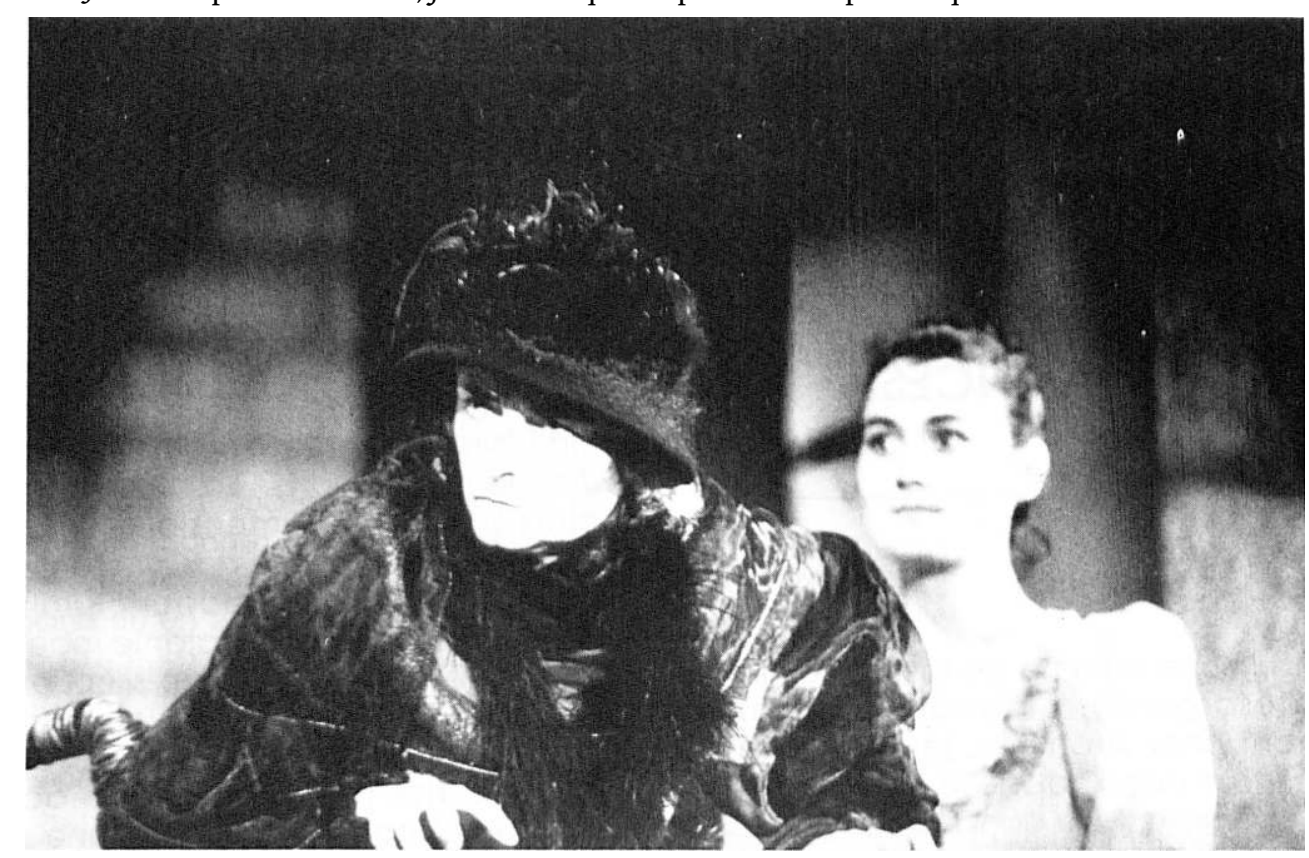

(C) Collectif Photo BVOJ 90.

\section{La Dispute (1744), comédie en un acte}

Changement de climat avec la deuxième pièce du spectacle. Par l'aménagement de l'espace d'abord: l'espace scénique est dans la salle, au niveau de l'espace du spectateur. Un grand rideau noir efface la scène surélevée du Grand-Kursaal, créant un milieu resserré et proche du public. Décor : une étendue de sable, une pyramide noire, un paravent sur la droite. Musique: La Dispute est accompagnée d'une musique originale de Manuel Polleti. Cette musique, ni accessoire, ni ornement, assure une fonction dramatique. La pièce est divisée en sept nuits et la musique raconte à chaque fois pendant la nuit ce qui va se dérouler le jour. Chaque personnage a sa couleur musicale grâce à un instrument qui le représente: clarinette pour Eglé, violoncelle pour Azor, bangas pour Carise...

\section{L'inconstance}

Qui de l'homme ou de la femme a donné le premier exemple d'inconstance? Pour le savoir, un prince a imaginé une situation quasi expérimentale : il a fait élever dans un lieu solitaire deux garçons et deux filles, chacun grandissant séparément dans l'ignorance des autres. Au moment où commence la pièce, ils ont dix-huit ans et vont 
être mis en liberté. Le prince et Hermiane observent ces êtres semblables à une page blanche qui vont faire l'apprentissage de la rencontre sociale avec l'autre :

Les premières amours vont recommencer - dit le prince - nous verrons ce qui en arrivera.

12 L'état d'innocence s'exprime dans la parole, les postures du corps, le vêtement. Le langage accompagne la découverte du monde; il faut nommer les choses nouvelles: cette eau qui roule à terre s'appelle un ruisseau, cet objet s'appelle un homme. Le rapport au corps suggère une liberté naturelle : animalité féline des corps sur le sable, vêtements à demi ouverts sur une nudité candide en contraste avec le prince et Hermiane enfermés dans leurs costumes compliqués de dentelles et de volants.

13 L'innocence pourtant ne résiste pas à la tentation de la nouveauté. L'infidélité, la possibilité même d'un amour durable est la question qui parcourt la pièce. La preuve est donnée, et doublement, que l'inconstance est partie de notre humaine nature : Eglé trompe Azor, Mesrin trompe Adine. Les spécialistes de Marivaux n'auront pas manqué de remarquer une coupure dans le texte de la scène finale. Pour ne pas finir sur un constat pessimiste, Marivaux introduit au dernier moment un autre couple chargé d'illustrer en quelques répliques la thèse de la fidélité. Ce didactisme a gêné J.-M. Potiron :

La thèse est tellement belle que mettre une antithèse de façon aussi stéréotypée, ce n'est plus de l'art... J'ai mis trois mois pour savoir comment j'allais terminer la pièce ; ça m'est venu au cours des quinze derniers jours, dans l'urgence... Certains diront que j'ai perverti le texte mais je ne regrette pas cette dernière image qui pose tant de questions.

14 Le spectacle se termine effectivement par l'image-tableau d'un couple symbolisant l'espoir, message mis en doute par un coup de faux de la musique. (N.B. : il ne sera rien dit de cette image afin de laisser au spectateur futur le plaisir de la découverte).

\section{Nature et culture}

15 L'inconstance n'est que le thème émergent d'une pièce qui en fait soulève un débat d'essence philosophique opposant nature et culture.

Dans ma réflexion, je me suis aidé d'un seul livre : Le Contrat Social de Rousseau. Dans l'histoire de l'humanité, l'homme à l'état de nature était une espèce d'animal, un barbare; ce à quoi Rousseau tente de s'opposer avec l'image du bon sauvage. L'éducation, l'école, la famille, la science ont travaillé à rendre le monde meilleur, ont essayé d'assurer le progrès de l'humanité. Ce que j'ai voulu montrer dans cette pièce, c'est que la culture conduit aussi à la barbarie, comme en 14-18 ou dans les camps de concentration. On peut accéder à la fin du monde à travers notre apprentissage ; c'est ce que je dis pendant une heure quinze.

De nombreux signes renvoient dans la scénographie à l'idée de mort et de fin du monde. Le sable, entre mer et terre, c'est l'aube du monde mais aussi son terme quand il ne reste que de la poussière de terre. La pyramide symbolise les débuts de la culture en Égypte au milieu du désert de sable et représente aussi la mort.

Je voulais mettre un côté tragique, finir par la tragédie alors j'ai mis un tombeau.

17 L'avant-dernière scène montre de façon violente les « failles » de la culture. Les «bons enfants", transformés en barbares, tuent leurs maîtres, les dépouillent de leurs vêtements. 
Les enfants sont des sortes de Frankenstein créés de toute pièce qui se retournent contre leurs créateurs. Se revêtir de leurs vêtements, c'est accéder à la culture, c'est dire "voilà ce qu'on peut être ». Je leur ai demandé d'entasser les cadavres du prince et d'Hermiane comme dans un camp de la mort. J'ai voulu montrer ce qu'on peut être...

\section{Le spectacle : un public divisé}

La réunion des deux pièces dans le même spectacle correspond pour le metteur en scène à un travail unitaire qu'il conçoit mal de séparer. Le public quant à lui semble s'être scindé en deux groupes: ceux qui ont aimé Les Fausses Confidences et pas La Dispute, et inversement, les partisans de La Dispute étant les plus nombreux. Beau retour des choses pour une pièce assez peu jouée et qui, à sa création en 1744, fut un échec total. La Dispute ne fut représentée qu'une seule fois, devant 571 spectateurs dit la chronique.

Le spectateur moderne est-il plus réceptif au thème de La Dispute, à la mise en scène plus "contemporaine" dans sa forme visuelle? Est-il moins attentif aux Fausses Confidences, abusé par la familiarité de signes externes (costumes, perruques) qui émoussent sa perception? Ceux qui veulent juger «sur pièces " pourront le faire fin septembre, mais en discontinu, Les Fausses Confidences et La Dispute étant données séparément.

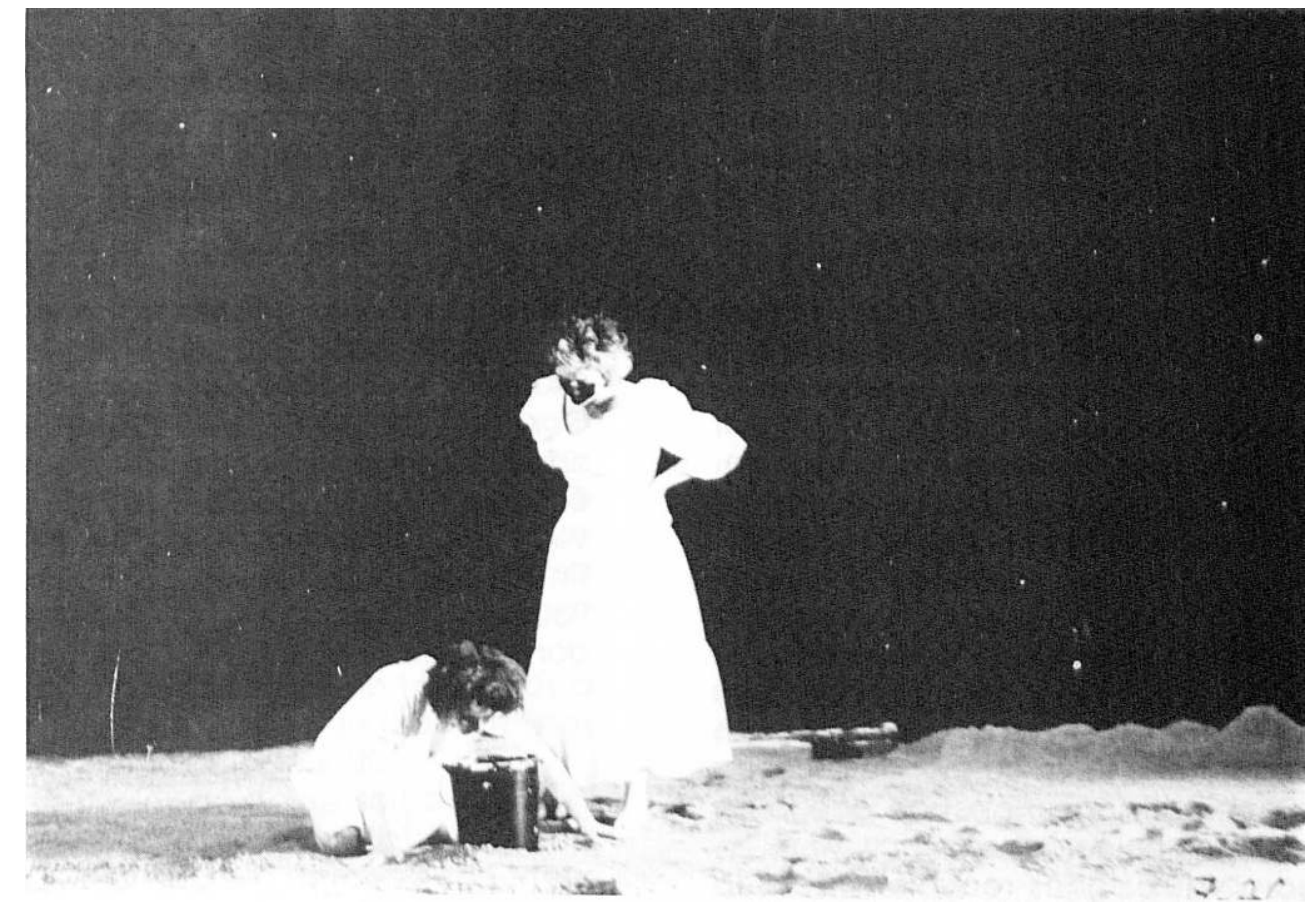

(c) Collectif Photo BVOJ 90. 